RESEARCH ARTICLE

\title{
High-power long-wave infrared laser based on polarization beam coupling technique
}

\author{
Yingjie Shen ${ }^{1}$, Chuanpeng Qian ${ }^{2}$, Xiaoming Duan ${ }^{3}$, and Ruijun Lan ${ }^{1}$ \\ ${ }^{1}$ School of Opto-electronic Information Science and Technology, Yantai University, Yantai 264005, China \\ ${ }^{2}$ Shanghai Institute of Optics and Fine Mechanics, Chinese Academy of Sciences, Shanghai 201800, China \\ ${ }^{3}$ National Key Laboratory of Tunable Laser Technology, Harbin Institute of Technology, Harbin 150001, China \\ (Received 25 November 2019; revised 17 January 2020; accepted 2 March 2020)
}

\begin{abstract}
We demonstrated a high-power long-wave infrared laser based on a polarization beam coupling technique. An average output power at $8.3 \mu \mathrm{m}$ of $7.0 \mathrm{~W}$ was achieved at a maximum available pump power of $107.6 \mathrm{~W}$, corresponding to an optical-to-optical conversion of $6.5 \%$. The coupling efficiency of the polarization coupling system was calculated to be approximately $97.2 \%$. With idler single resonance operation, a good beam quality factor of $\sim 1.8$ combined with an output wavelength of $8.3 \mu \mathrm{m}$ was obtained at the maximum output power.
\end{abstract}

Keywords: long-wave infrared laser; optical parametric amplifier; optical parametric oscillator; polarization coupling

\section{Introduction}

High-power long-wave infrared laser sources, operating most prominently in the region $8-12 \mu \mathrm{m}$ transparency window of the atmosphere, are currently of great interest in a variety of remote-sensing, lidar, medical surgery, defense and civilian applications and fundamental scientific research ${ }^{[1,2]}$. These lasers are the key components in directive systems for countermeasures against infrared sensors. They can also be used to identify the 'molecular fingerprint', which could supply scientific genetic classification for plant germplasm $^{[3]}$.

Since zinc germanium phosphide $\left(\mathrm{ZnGeP}_{2}, \mathrm{ZGP}\right)$ has a high nonlinear optical coefficient $d_{\mathrm{eff}}=75 \mathrm{pm} / \mathrm{V}$ and an optical transmission range of $2-12 \mu \mathrm{m}^{[4]}$, ZGP-based optical parametric oscillators (OPOs) and optical parametric amplifiers (OPAs) pumped by $2 \mu \mathrm{m}$ pulsed lasers are the most attractive $8-12 \mu \mathrm{m}$ sources. Zinc germanium phosphide, offering excellent optical, mechanical and thermal properties, is also commercially available at present. In a general way, the OPO cavity configuration consists of a plane-plane cavity (standing-wave oscillator) ${ }^{[5-7]}$, a Vshaped three-mirror ring resonator ${ }^{[8]}$ and a four-mirror ring resonator (traveling-wave cavity) ${ }^{[9-11]}$. The OPO consists of

Correspondence to: Y. Shen, School of Opto-electronic Information Science and Technology, Yantai University, Yantai 264005, China. Email: yingjieyj@163.com two operating modes: single resonance (SRO) and double resonance (DRO). Single resonance means that only one kind of parametric light oscillates in the cavity. By definition, DRO means that the signal and idler lights oscillate in the cavity.

The most challenging component of the system is the efficient $2 \mu \mathrm{m}$ pulsed laser. Both bulk Ho lasers and Ho fiber lasers allowing $Q$-switched operation with high pulse energies can be suitable for pumping a ZGP OPO or OPA ${ }^{[12-14]}$. The second challenge is to reduce the effect of the feedback from the OPO or the OPA into the pump laser and the risk of the damage to the mirror or medium coating and the nonlinear medium. An efficient $2 \mu \mathrm{m}$ pump source was reported in our previous work ${ }^{[15]}$. In order to generate high output power, besides the pump source, some novel solutions must be used to avoid optical damage of the ZGP crystal of the order of $1-2 \mathrm{~J} / \mathrm{cm}^{2}$. Our goal in this work is to address the above matter. We divided the nanosecond pulsed Ho laser into two parts with different polarization states. One of the two beams with a slightly higher energy was used to pump a four-mirror ring OPO and a cascaded OPA and the other one to pump a linear OPO. The two beams passed through a thin polarization plate and formed an integrated laser. This is a realistic solution for improving the output energy of a long-wave laser without any damage. This application can be extended to other high-power lasers.

In this paper, we demonstrated a high-power longwave infrared laser based on a polarization beam coupling 
technique. An average output power of $7.0 \mathrm{~W}$ was achieved at a maximum available pump power of $107.6 \mathrm{~W}$, corresponding to an optical-to-optical conversion of $6.5 \%$. This is a feasible method to increase the output power of longwave infrared lasers without any damage.

\section{Working principle}

Zinc germanium phosphide was selected for the long-wave laser in our experiment because of its excellent physical properties. The absorption coefficient of the ZGP crystal (School of Chemical Engineering \& Technology, HIT) at $2.09 \mu \mathrm{m}$ was measured to be $0.03 \mathrm{~cm}^{-1}$. The Sellmeier equations of the ZGP crystal $(\lambda$ in $\mu \mathrm{m}, 1.5 \mu \mathrm{m}<\lambda<$ $12 \mu \mathrm{m}, T=293 \mathrm{~K})$ are as follows ${ }^{[16]}$ :

$$
\begin{aligned}
& n_{o}^{2}=8.0409+\frac{1.68625 \lambda^{2}}{\lambda^{2}-0.40824}+\frac{1.288 \lambda^{2}}{\lambda^{2}-611.05}, \\
& n_{e}^{2}=8.0929+\frac{1.8649 \lambda^{2}}{\lambda^{2}-0.41468}+\frac{0.84052 \lambda^{2}}{\lambda^{2}-452.05} .
\end{aligned}
$$

According to Equation (1), the indexes of refraction at the principal axis, $n_{\mathrm{o}}=3.1451$ and $n_{\mathrm{e}}=3.1852$, were calculated for $2.09 \mu \mathrm{m}$. The difference of the two indexes is about 0.04 , caused by birefringence, which introduces a walk-off effect in the nonlinear crystal.

As shown in Figure 1, phase-matching curves of ZGP were calculated by using the refractive index ellipsoid equation for a uniaxial crystal combined with energy conservation $\left(1 / \lambda_{p}=1 / \lambda_{s}+1 / \lambda_{i}\right)$ and momentum conservation equations $(\Delta k=0)^{[17]}$. In the $2.09 \mu \mathrm{m}$ pump laser for typeI phase matching, when the idler wavelength is $8000 \mathrm{~nm}$, the phase-matching angle is $51.2^{\circ}$, corresponding to a signal of wavelength $2846 \mathrm{~nm}$. When the idler wavelength is $8300 \mathrm{~nm}$, the phase-matching angle is $51.0^{\circ}$, which corresponds to a signal of wavelength $2832 \mathrm{~nm}$. In the $2.09 \mu \mathrm{m}$ pump laser for type-II phase matching, when the idler wavelength is $8000 \mathrm{~nm}$, the phase-matching angle is $65.0^{\circ}$, corresponding to a signal of wavelength $2835 \mathrm{~nm}$. When the idler wavelength is $8300 \mathrm{~nm}$, the phase-matching angle is $63.9^{\circ}$, which corresponds to a signal of wavelength $2807 \mathrm{~nm}$. From a tuning of $8000 \mathrm{~nm}$ to $8300 \mathrm{~nm}$, the angle of type I needed to be rotated only $0.2^{\circ}$, while the type-II angle is rotated up to $1.1^{\circ}$. Obviously, the type-I phase matching has greater tuning sensitivity than the type-II one.

Figure 2 shows the schematic diagram of the setup of a farinfrared laser with the polarization beam coupling technique. The system consists of one rectangular ring resonator OPO (as shown in Figure 2(b)) cascaded with an OPA (as shown in Figure 2(c)), a linear plane-plane cavity OPO (as shown in Figure 2(a)) and a pulsed laser as the only pump source. The pulsed Ho laser (average output power of $107.6 \mathrm{~W}$, pulse width of $30 \mathrm{~ns}$ and pulse repetition rate of $18 \mathrm{kHz}$ ) was

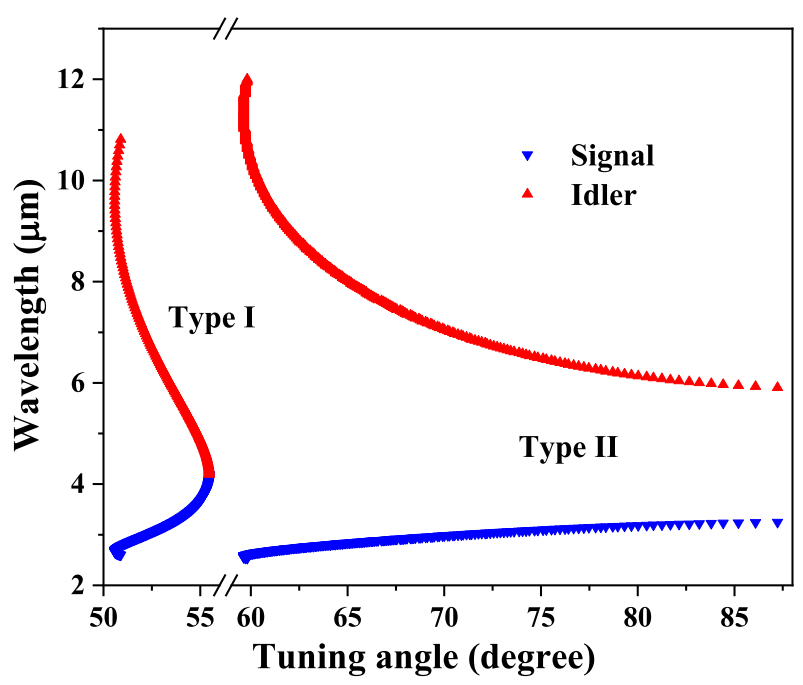

Figure 1. ZGP crystal angular tuning curves for type-I and type-II phase matching.

divided into two parts with different polarizations, which is the critical factor of the coupling technique, by a halftransmission and half-reflection plane mirror M1 (exactly $44.3 \%$ reflectivity and $55.7 \%$ transmission at $2.09 \mu \mathrm{m}$ ).

The linear resonator consists of two flat mirrors M7 (coating with high transmittance at $2.09 \mu \mathrm{m}$ and high reflectivity at 8.0-8.4 $\mu \mathrm{m}$ ) and M8 (coating with $27 \%$ transmittance at $8.0-8.4 \mu \mathrm{m}$ ). In order to improve the beam quality of the long-wave laser, SRO was used in the linear OPO resonator. The flat mirrors M9 and M10 have high transmittance at $8.0-8.4 \mu \mathrm{m}$ and high reflectivity at $2.8 \mu \mathrm{m}$ and $2.09 \mu \mathrm{m}$, respectively. In this end-pumped or longitudinal pump scheme, the pump beam and the parametric laser propagate in the same direction. The angle of incidence on the cavity mirrors (M7 and M8) was approximately $3^{\circ}$. An aperture was used to avoid the pump laser feedback from the input mirror and the end face of the nonlinear material, as shown in Figure 2(a). The physical length of the linear cavity was $30 \mathrm{~mm}$. The ZGP2 crystal was located at the center of the resonator.

Figure 2(b) shows a typical four-mirror rectangular cavity, consisting of four plane mirrors and a nonlinear medium. The primary characteristic of this structure is that the pumping beam and the parametric light always propagate in the same direction, suppressing the standing-wave effect and spatial hole-burning effect in the resonator effectively. The stability of the ring cavity is good; even though the focal length of the thermal focal lens changes dramatically, the resonant cavity can still be in a stable region. Especially under the condition of high-power pump, the ring cavity still has high stability. In addition, the greater physical length of the ring cavity results in good beam quality. In our experiment, the ring OPO cavity includes four plane $45^{\circ}$ mirrors. The third mirror M3 was coated for high transmittance at $2.09 \mu \mathrm{m}$ and high reflectivity at $8.0-8.4 \mu \mathrm{m}$. The last mirror M4 was an output coupler coated for $27 \%$ transmission 


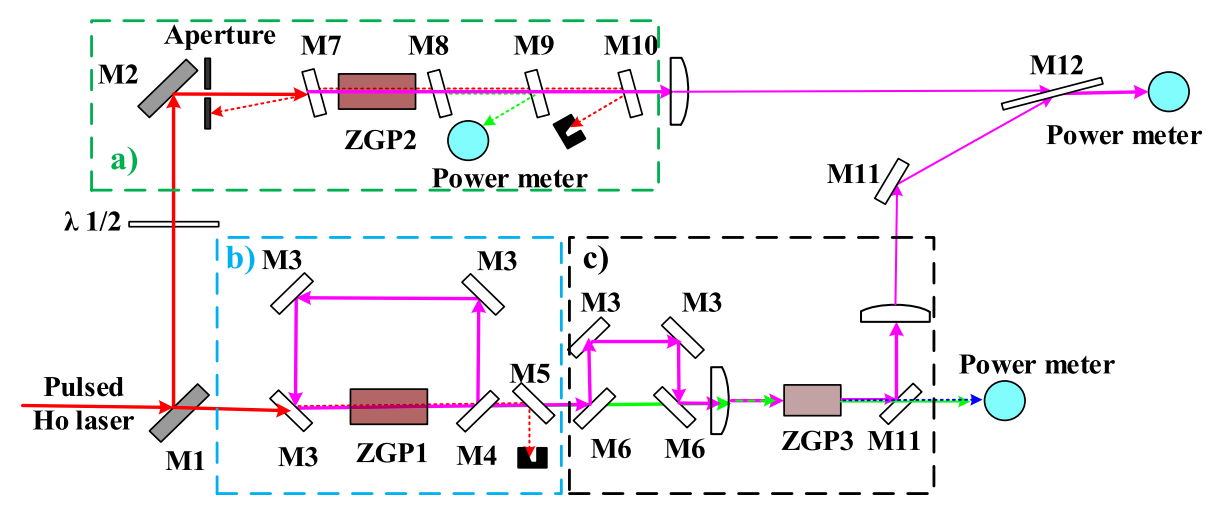

Figure 2. Schematic diagram of the far-infrared laser system: (a) linear OPO resonator; (b) ring OPO cavity; (c) OPA system.

at $8.0-8.4 \mu \mathrm{m}$. The flat $45^{\circ}$ mirror M5, coated for high transmittance at the signal and the idler and high reflectivity at the pump laser, was used to separate the signal and the idler from the residual pump laser. The physical length of the ring cavity was $102 \mathrm{~mm}$. The ZGP1 crystal was located at the middle of mirrors $\mathrm{M} 3$ and M4.

In the OPO, ZGP1 and ZGP2, with a dimension of $6 \mathrm{~mm} \times$ $6 \mathrm{~mm} \times 25 \mathrm{~mm}$, were all cut for type-I phase matching $(\theta=$ $51.5^{\circ}$ and $\varphi=0^{\circ}$ relative to the optical axis). The ZGP3 cut for type-II phase matching $\left(\theta=68.4^{\circ}\right.$ and $\varphi=45^{\circ}$ relative to the optical axis) has a dimension of $6 \mathrm{~mm} \times 6 \mathrm{~mm} \times 24 \mathrm{~mm}$. In that case, the two parametric beams with two different polarizations can be recombined into one beam by a thin polarization plate.

Due to the walk-off effect in the nonlinear crystal, a beam coincidence system was used to separate and overlap the signal and the idler from the OPO before focusing into the OPA crystal. The system consists of four plane $45^{\circ}$ mirrors. Two of them were the same as M3; the other two M6 mirrors were coated for high reflectivity at $8.0-8.4 \mu \mathrm{m}$ and high transmittance at $2.6-2.8 \mu \mathrm{m}$. The $8.3 \mu \mathrm{m}$ laser from the OPA was separated by two dichroic mirrors M11 coated for high reflectivity at $8.0-8.4 \mu \mathrm{m}$ and high transmittance at $2.6-2.8 \mu \mathrm{m}$ and $4.0-4.3 \mu \mathrm{m}$. The pump beam diameter for pumping the ZGP crystal was usually focused to be $0.6-$ $1.1 \mathrm{~mm}$ from the threshold to the maximum output. The dimension of the pump beam diameter is not only to satisfy the pump intensity of the OPO and OPA but also to avoid coating damage and deep damage of the nonlinear crystal.

Obviously, the idler beam (wavelength above $8 \mu \mathrm{m}$ ) is our parametric light target. For the OPO, the idler (extraordinary light) and signal lasers (extraordinary light) are all different from the pump laser (ordinary light) because of the type-I phase matching. When the idler laser is used as the seed laser and the signal laser as the pump beam, the ZGP3 crystal can be anything but type-II phase matching since the idler laser (seed laser of OPA, extraordinary light) and the signal laser (pumping beam of the OPA, extraordinary light) propagate along the same polarization, and the other parametric beam travels in a different polarization direction (ordinary light).
Besides the OPA, another feasible method to increase the output power or energy of a far-infrared laser is to employ a polarization beam coupling technique since the parametric beams are all polarized. Because of the low coating damage threshold $\left(\sim 1 \mathrm{~J} / \mathrm{cm}^{2}\right)$ of nonlinear crystal films and mirrors, the polarization coupling technique is a possible solution to increase the output power of farinfrared lasers. The most crucial factor in this system is the coupling efficiency of the polarizer since the linewidth of the far-infrared laser from the OPO is very large. Another key factor is that the two different polarized lights must be focused into the same spot size on the polarizer by a focusing lens system. Apparently, the configuration of this design is very large, which cannot be applied in a system of compact and lightweight configuration. The flat mirror M12 is a polarization beam splitter plate. The parallel polarized $8.3 \mu \mathrm{m}$ laser from the linear OPO resonator can pass through the mirror M12 at a certain angle. However, the vertical polarized $8.3 \mu \mathrm{m}$ laser from the OPA system can be reflected from M12.

\section{Results and discussion}

In the above configuration, as shown in Figure 2(b), the maximum output power at $8.3 \mu \mathrm{m}$ was $3.5 \mathrm{~W}$ when pumped with $59.9 \mathrm{~W}$ of $2.1 \mu \mathrm{m}$ pump laser, corresponding to an average $2.8 \mu \mathrm{m}$ laser output power of $14.4 \mathrm{~W}$, as shown in Figure 3. The conversion efficiency and slope efficiency from $2.1 \mu \mathrm{m}$ to $8.3 \mu \mathrm{m}$ were about $5.84 \%$ and $10.3 \%$, and those from $2.1 \mu \mathrm{m}$ to $2.8 \mu \mathrm{m}$ were approximately $24.0 \%$ and $41.5 \%$, respectively. After passing through the separated and recombined system (as shown in Figure 2(c)), the average powers for $8.3 \mu \mathrm{m}$ and $2.8 \mu \mathrm{m}$ lasers were $3.3 \mathrm{~W}$ and $9.6 \mathrm{~W}$, respectively. When they were focused into the ZGP3 crystal, as shown in Figure 3, the average output power of the $8.3 \mu \mathrm{m}$ laser was increased to $4.7 \mathrm{~W}$. No coating damage and crystal damage were observed in the whole experiment. With no increase in wavelength $2.1 \mu \mathrm{m}$ for the pump laser in the OPA 


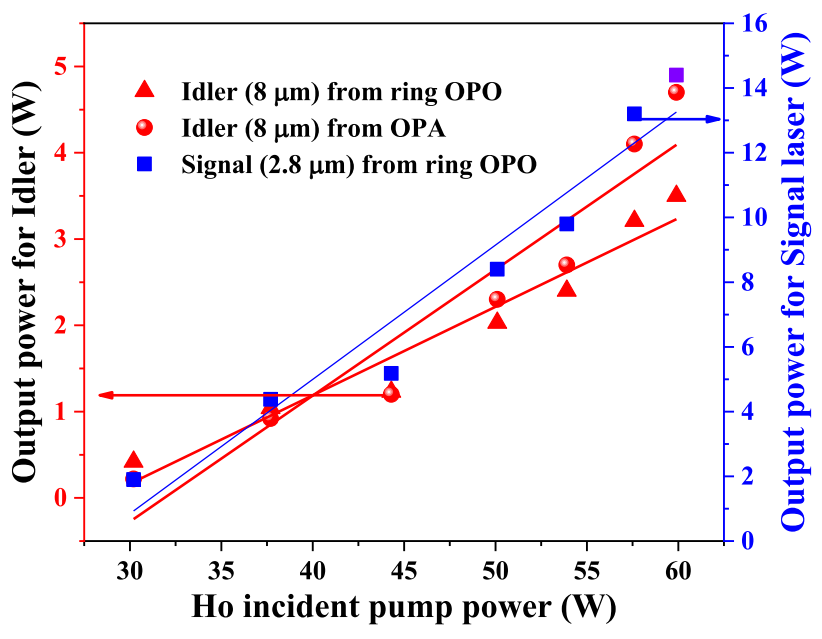

Figure 3. Output performance of the ring OPO and cascaded OPA system.

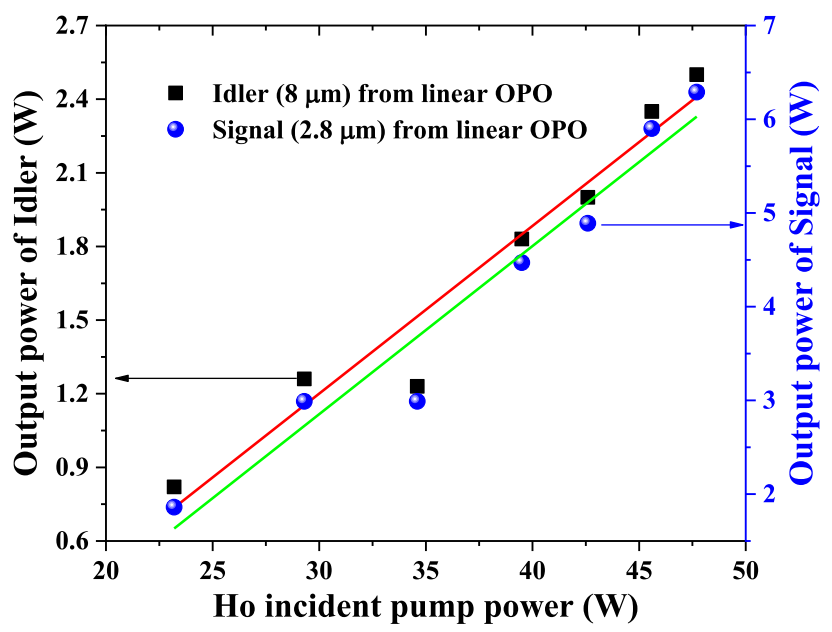

Figure 4. Output performance of the linear OPO.

system, the $8.3 \mu \mathrm{m}$ laser was up to $42.4 \%$ from the output of the OPO.

In the above system, as shown in Figure 2(a), the maximum output powers for $8.3 \mu \mathrm{m}$ and $2.8 \mu \mathrm{m}$ from the linear cavity were about $2.5 \mathrm{~W}$ and $6.3 \mathrm{~W}$ at a Ho incident pump power of $47.7 \mathrm{~W}$, respectively, as shown in Figure 4. The optical-to-optical conversion efficiency and slope efficiency from $2.09 \mu \mathrm{m}$ to $8.3 \mu \mathrm{m}$ of $5.24 \%$ and $6.8 \%$ were achieved, which were slightly smaller than those in the ring OPO cavity. The optical conversion efficiency and slope efficiency from $2.09 \mu \mathrm{m}$ to $2.8 \mu \mathrm{m}$ were about $13.2 \%$ and $17.9 \%$, which were much lower than those in the ring OPO cavity. The reason for this is probably that the standing wave $(8.3 \mu \mathrm{m})$ in the linear OPO cavity limits the output of the signal laser $(2.8 \mu \mathrm{m})$.

After passing through or being reflected from the thin polarizer, the coupling output power from the polarizer was up to $7.0 \mathrm{~W}$, as shown in Figure 5, corresponding to a coupling efficiency of $97.2 \%$ with respect to the sum

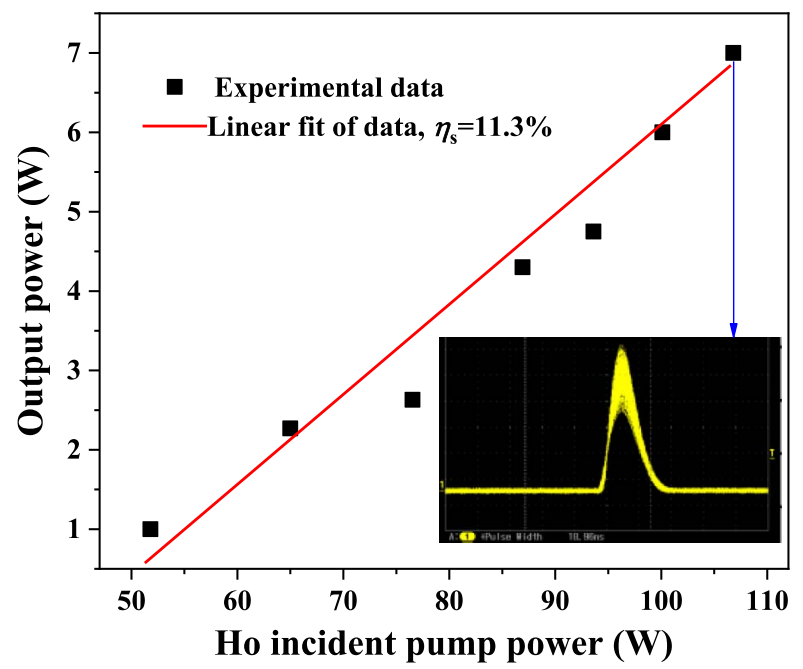

Figure 5. Output performance with the use of a thin polarized plate; inset shows an oscilloscope profile at the maximum output power.

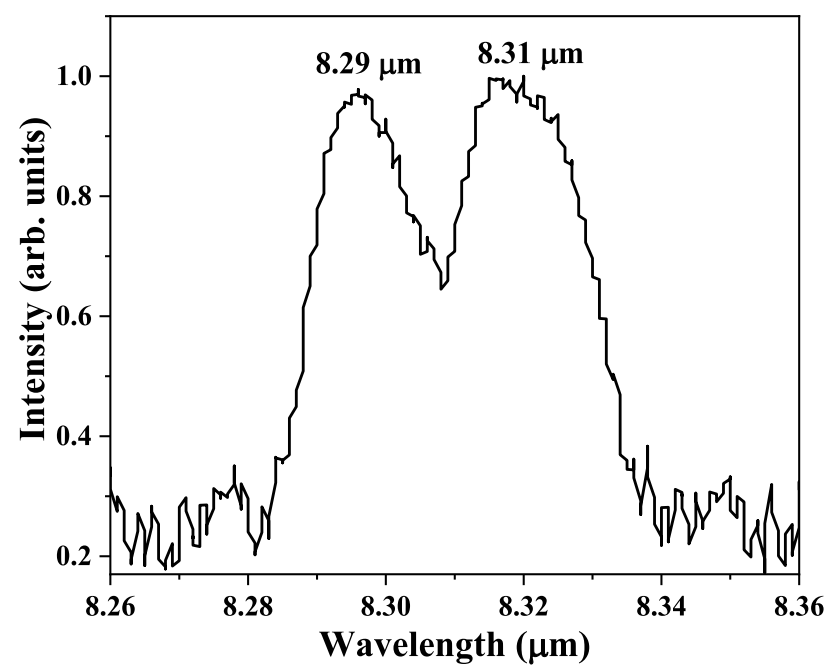

Figure 6. Output spectrum of the far-infrared laser.

of $2.5 \mathrm{~W}$ and $4.7 \mathrm{~W}$. The shortest pulse width was about $19.0 \mathrm{~ns}$ at a Ho incident pump power of $107.6 \mathrm{~W}$, the typical oscilloscope trace of which is shown in the inset of Figure 5. Although the spectral linewidth of the far-infrared laser from the OPO or the OPA was very large, the coupling efficiency of the polarizer was not as low as expected. The above excellent results proved that the polarization beam coupling technique can be successfully employed in a high-power farinfrared laser from the OPO or the OPA. No coating damage was observed in the whole experiment, implying that it is an efficient and dependable design.

The output spectrum of the $8.3 \mu \mathrm{m}$ laser from the above system, as shown in Figure 6, was measured by a $150 \mathrm{~mm}$ WDG30-Z monochromator combined with a HgCdTe detector. We have conducted spectrum measurements several times, which takes 15 min each. All of them were observed 


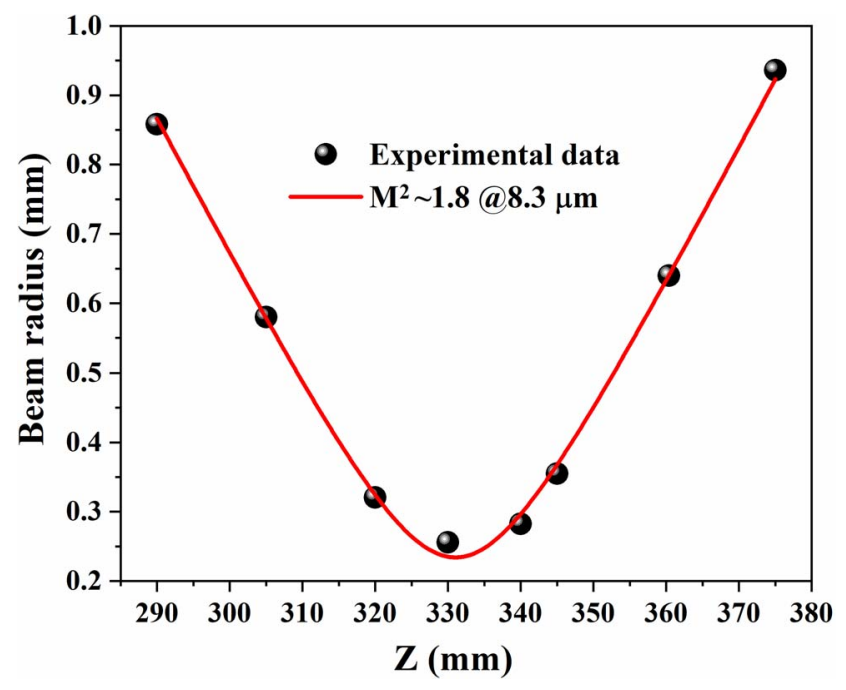

Figure 7. The beam quality of the far-infrared laser.

as the same profile, implying that the high-power system lies in a stable operating region. The spectra range from $8283 \mathrm{~nm}$ to $8336 \mathrm{~nm}$ and have two slightly different peaks at $8290 \mathrm{~nm}$ and $8310 \mathrm{~nm}$, which can be probably attributed to the small difference in phase-matching angles of the two parts from the polarization beam combined system.

To evaluate the beam quality of the $8.3 \mu \mathrm{m}$ laser, by using a ZnSe focal lens with a $200 \mathrm{~mm}$ focusing length, the beam radius at the highest output power was measured by the 90/10 knife-edge technique. The propagation characteristics of the $8.3 \mu \mathrm{m}$ laser at the highest output power of $7.0 \mathrm{~W}$ are shown in Figure 7. By fitting these experimental data according to the Gaussian propagation equation, the quality beam factor $M^{2}$ was about 1.8 at the output wavelength of $8.3 \mu \mathrm{m}$, which was smaller than 3.6 obtained by the signal from a singly resonant oscillator ${ }^{[18]}$ and 2.7 from a doubly resonant oscillator ${ }^{[19]}$. This proves that the idler SRO can really improve the beam quality compared with the previous report.

\section{Conclusion}

In this paper, the scheme to increase output power is suitable for long-wave infrared lasers since the signal beam of the ring OPO is the ideal pumping source for a cascaded OPA laser and the long-wave signal beam can reduce the quantum loss of the OPA. If the two arms of the coupling system are all ring OPOs and cascaded OPAs, the output power and conversion efficiency from $2.1 \mu \mathrm{m}$ to $8.3 \mu \mathrm{m}$ of the system will attain higher values. The high coupling efficiency proves that a system with a thin polarization plate is a feasible solution to increase the output power or energy of $8.3 \mu \mathrm{m}$.
Furthermore, the mid-infrared laser can achieve good output performance without using the beam coupling technique due to its high damage threshold and quantum efficiency.

\section{Acknowledgement}

This work was supported by the National Natural Science Foundation of China (NFSC) (Nos. 61805209 and 51572053).

\section{References}

1. D. L. Snavely and J. Dubsky, J. Polymer Sci. A 34, 2575 (1996).

2. Y. He, P. Wang, R. T. White, and B. J. Orr, Opt. Photonics News 13, 56 (2002).

3. C. R. Petersen, U. Møller, I. Kubat, B. Zhou, S. Dupont, J. Ramsay, T. Benson, S. Sujecki, N. Abdel-Moneim, Z. Tang, D. Furniss, A. Seddon, and O. Bang, Nat. Photonics 8, 830 (2014).

4. E. C. Cheung, S. Palese, H. Injeyan, C. Hoefer, J. Ho, R. Hilyard, H. Komine, J. Berg, M. M. Fejer, H. Injeyan, and U. Keller, in Advanced Solid State Laser (1999), paper WC1.

5. A. Hemming, J. Richards, A. Davidson, N. Carmody, S. Bennetts, N. Simakov, and J. Haub, Opt. Express 21, 10062 (2013).

6. E. O. Ammann, M. K. Oshman, J. D. Foster, and J. M. Yarborough, Appl. Phys. Lett. 15, 131 (1969).

7. Hemming, J. Richards, A. Davidson, N. Carmody, S. Bennetts, N. Simakov, P. Davies, and J. Haub, in International Quantum Electronics Conference \& Conference on Lasers and ElectroOptics-Pacific Rim (2011), paper C607.

8. E. Lippert, H. Fonnum, G. Arisholm, and K. Stenersen, Opt. Express 18, 26475 (2010).

9. G. Stoeppler, N. Thilmann, V. Pasiskevicius, A. Zukauskas, C. Canalias, and M. Eichhorn, Opt. Express 20, 4509 (2012).

10. A. Hemming, J. Richards, A. Davidson, N. Carmody, N. Simakov, M. Hughes, P. Davies, S. Bennetts, and J. Haub, in Conference on Lasers and Electro-Optics (2013), paper CW1B.7.

11. P. G. Schunemann, in Conference on Lasers and ElectroOptics/Quantum Electronics and Laser Science Conference and Photonic Applications Systems Technologies (2007), paper CThL3.

12. A. Dergachev, D. Armstrong, A. Smith, T. Drake, and M. Dubois, Opt. Express 15, 14404 (2007).

13. C. Kieleck, A. Berrou, B. Donelan, B. Cadier, T. Robin, and M. Eichhorn, Opt. Lett. 40, 1101 (2015).

14. M. Gebhardt, C. Gaida, P. Kadwani, A. Sincore, N. Gehlich, C. Jeon, L. Shah, and M. Richardson, Opt. Lett. 39, 1212 (2014).

15. Y.-J. Shen, B.-Q. Yao, X.-M. Duan, G.-L. Zhu, W. Wang, Y.-L. $\mathrm{Ju}$, and Y.-Z. Wang, Opt. Lett. 37, 3558 (2012).

16. D. N. Nikogosyan, Nonlinear Optical Crystals: A Complete Survey (Springer Science Business Media, Inc., 2005).

17. R. W. Boyd, Nonlinear Optics (Academic Press, 2008).

18. M. W. Haakestad, G. Arisholm, E. Lippert, S. Nicolas, G. Rustad, and K. Stenersen, Proc. SPIE 6998, 699812 (2008).

19. E. Lippert, G. Rustad, G. Arisholm, and K. Stenersen, Opt. Express 16, 13878 (2008). 